\title{
Adjustment to the Light Element Areal Concentration Calculation for Neutron Depth Profiles
}

Jamie Weaver ${ }^{1}$ and Anna Job ${ }^{2}$

${ }^{1}$ NIST, Material Measurement Laboratory, United States, ${ }^{2}$ National Institute of Standards and Technology, United States

Neutron-based techniques are well-suited for the quantification of light elements due to neutron interactions with atomic nuclei. This contrasts with $\mathrm{x}$-ray or ion-based techniques which relay on electron shell interactions for their detection mechanisms, and, as a result, may be less sensitive to light (e.g., low $\mathrm{Z}$ ) elements. Of the neutron techniques available for light element detection, neutron depth profiling (NDP) is popular for the measurement of $\mathrm{Li}, \mathrm{B}, \mathrm{He}$, and N [1, 2]. Since its inception in 1968 by J. P. Biersack [3], NDP measurements have made significant impacts in the industrially important fields of energy materials (e.g., Li-ion cells, [4]), semiconductors (e.g., B doped Si, [5]), and nuclear materials research (e.g., He diffusion in proposed fusion reactor materials, [6]). The utility of NDP is expected to increase over the coming decades with the continued rise in demand for new, more efficient Li-ion battery technologies [7] and innovation of next generation B doped semiconductors for quantum computing devices [8].

Several key challenges of NDP must be overcome to scale with this growing demand, including the development of more advanced and approachable data analysis methodology. A needed improvement is a reassessment of how NDP data is modeled and how those models are applied during data processing steps. Traditional NDP data analysis methods are centered on calculating stopping range table(s) with Stopping Range of Ions in Matter (SRIM) [9] for the construction of energy-to-depth calibration curves. This method, while providing accurate results [10,11], does not fully account for the effect of ion straggling - a significant phenomenon of charged particle transport that is important to accurately estimating NDP related uncertainties. The straggling can also influence the number of ions transmitted to the detector. This latter issue is the topic of this presentation.

The core-reaction of NDP is the capture of a cold or thermal neutron by a select isotope (e.g., ${ }^{6} \mathrm{Li},{ }^{10} \mathrm{~B}$, ${ }^{3} \mathrm{He}$, etc...) resulting in the formation of an unstable compound nucleus and the subsequent expulsion of two or more energetic charged particles (reaction products) with initial, characteristic energies. These energetic particles lose energy relative to the material's stopping force as they move through the material. Stopping force $\left(S_{f}\right.$, historically called the stopping power) is defined as the mean energy loss $(\Delta \mathrm{E})$ of an ion per collision over the mean free flight path between collisions $(\lambda)$ of the ion (Equation 1, [9]):

$$
\text { Equation } 1 \quad S_{f}=\Delta \mathrm{E} / \lambda
$$

he $S_{f}$ is material specific and has both electronic and nuclear components [11]. The magnitude of these components vary with increasing distance traveled by an ion. The nuclear component contributes more to the total $S_{f}$ of a material than the electronic component and it accounts for energy loss and deflection events due to atom-to-atom interaction between the ion and host material atoms. Between these events the ion can undergo electronic and nuclear energy straggling along an ion's free flight path (see Figure 1a). All these interactions are probabilistic, and it is expected that a range of exit energies and positions are possible for an ion generated at a singular origin position (Figure 1a). Stopping force theory also suggests that the total number of scattered ions increases with increased ion depth origin due the increased likelihood of an ion undergoing one or more of the above listed interactions. Regarding NDP experiments, this indicates that the deeper an ion originates within a sample the less likely it is to reach the detector. 
This factor is currently not covered by traditional NDP data processing methods as stopping range tables do not full account for energy straggling [11].

This gap can be overcome by constructing NDP energy-to-depth calibration curves from Monte Carlo simulations that include more accurate energy straggling calculations. To show proof-of-concept a simple Monte Carlo simulation of ion transport through a host material is constructed using the TRansport of Ions in Matter (TRIM), [9]) is constructed. A low $n$ (1E+4 ions) example of this work is herein presented for triton $\left({ }^{3} \mathrm{H}\right)$ ions, produced from the $n,{ }^{6} \mathrm{Li}$ reaction, continuously passing through variable thicknesses of $\mathrm{LiF}, 7.6 \mu \mathrm{m}$ polyimide film, and a vacuum distance representative of NDP chamber geometries (Figure 1c). This system is representative of one often encountered in battery related NDP experiments [4] . Calculated data is analyzed for total ions missing and hitting the NDP detector in the $\mathrm{y}$ (lateral) and $\mathrm{z}$ (longitudinal) directions as a function of ion origin depth. From these results a detected ion (assuming $100 \%$ detector efficiency) vs. depth calibration curve is created and fit using a linear model. Calculated constants for the fit are utilized as a correction to the traditional NDP counts-to-atom concentration equation (Figure 1d).

Results from these calculations will be presented along with an assessment of two important findings of the study: 1) a correction may need to be applied to the traditional NDP counts-to-areal concentration equation to account for ion loss as a function of origin depth and material type, and 2) there is an increase in the exit energy range of the ions as a function of depth. The second item, which has been previously discussed [12], indicates that the energy resolution of neutron depth profiles decreases with increasing ion origin depth; a factor that will also be important when estimating NDP

uncertainties. Future work will focus on rerunning the models assuming a 3D source rather than a point source and with a larger $n$ that is comparable to the neutron fluence rate for a cold neutron beam (e.g., $\approx 1 \mathrm{E}+9$ neutrons $\mathrm{cm}-2 \mathrm{~s}-1$ ).

The authors would like to acknowledge the NIST Summer High School Internship program which provided partial support for A. Job's portion of this work. Contributions of NIST are not subject to copyright. 


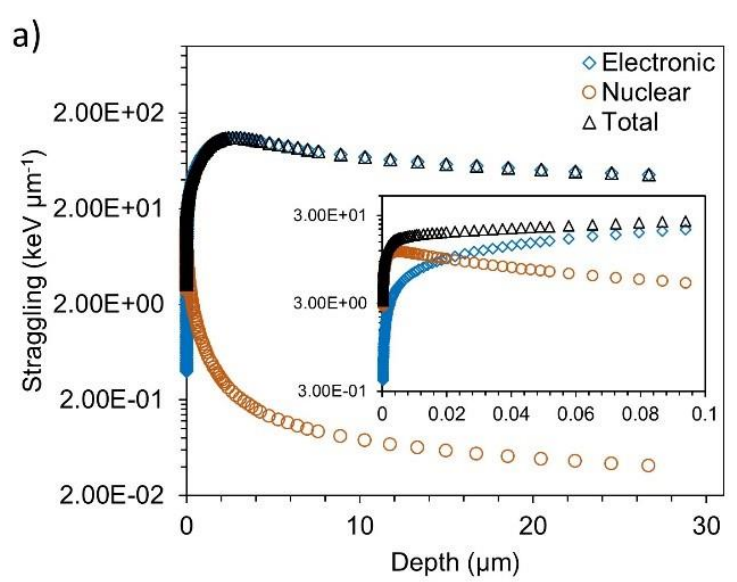

b)

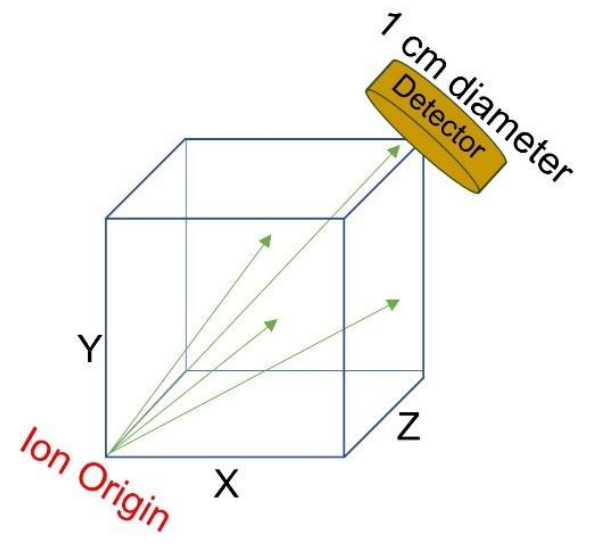

c)
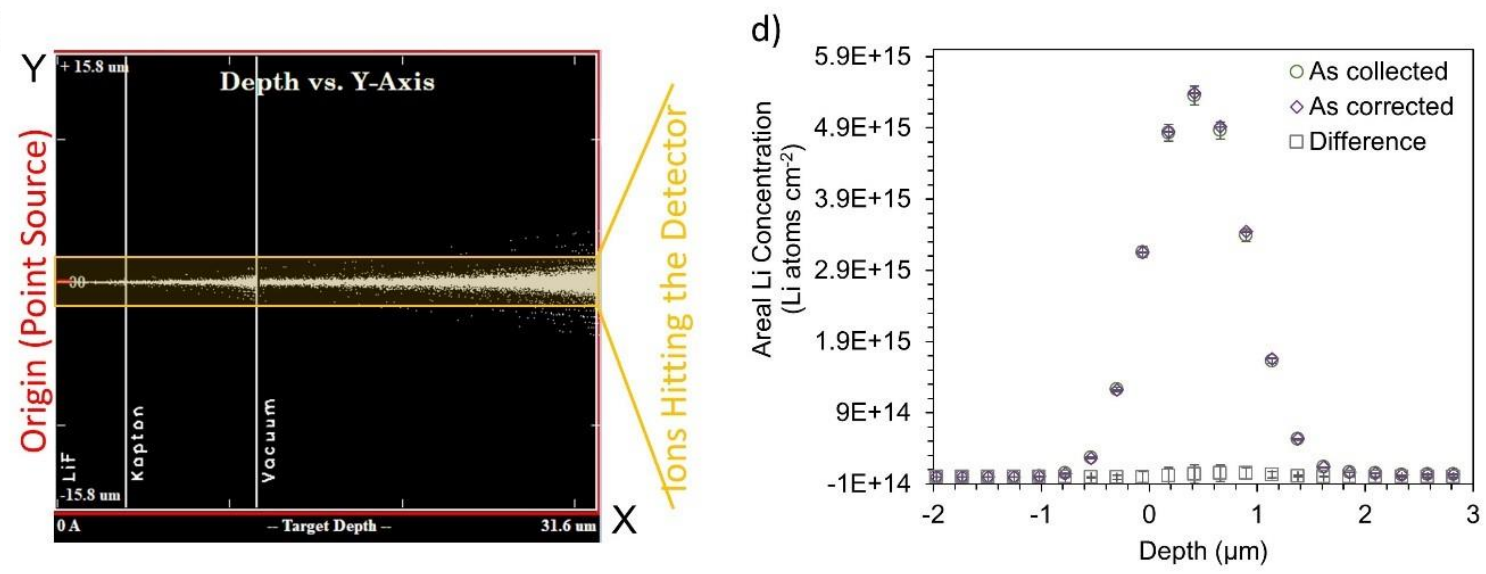

Figure 1. a) Simulated electronic and nuclear straggling components for a $3 \mathrm{H}$ ion passing through Kapton. b) laboratory frame of reference diagram for an ion's probable flight paths as it passes through a material and either hitting or missing an NDP detector. c) TRIM generated Monte Carlo simulation of $3 \mathrm{H}$ ions originating from $3 \mu \mathrm{m}$ deep in $\mathrm{LiF}$ and passing through $7.6 \mu \mathrm{m}$ of Kapton and several $\mu \mathrm{m}$ of vacuum (note the vacuum distance was trunked in this image for display reasons only; $20 \mathrm{~mm}$ of vacuum distance is utilized in the models for this study). d) As collected, traditionally processed, corrected, and difference NDP profiles of $1 \mu \mathrm{m}$ thick LiF film. Uncertainties are shown to $1 \sigma$ and based on experimental counting statistics.

\section{References}

1. Downing, R.G. Neutron Depth Profiling Chambers, Reactions of Interest. [Web page] 2018 Oct 24, 2018, 9:51 AM [cited 2018 Nov. 5]; Available from: https://sites.google.com/site/nistndp/home.

2. Lamaze, G.P., et al., Neutron depth profiling with the new NIST cold neutron source. Surface and Interface Analysis: An International Journal devoted to the development and application of techniques for the analysis of surfaces, interfaces and thin films, 1997. 25(3): p. 217-220.

3. Biersack, J., et al., The use of neutron induced reactions for light element profiling and lattice localization. Nuclear instruments and Methods, 1978. 149(1-3): p. 93-97.

4. Nagpure, S.C., et al., Neutron depth profiling technique for studying aging in Li-ion batteries.Electrochimica Acta, 2011. 56(13): p. 4735-4743. 
5. Downing, R., et al., The measurement of boron at silicon wafer surfaces by neutron depth profiling. MRS Online Proceedings Library Archive, 1989. 166.

6. Gilliam, S., et al., Retention and surface blistering of helium irradiated tungsten as a first wall material. Journal of nuclear materials, 2005. 347(3): p. 289-297.

7. $\quad$ Nitta, N., et al., Li-ion battery materials: present and future. Materials today, 2015. 18(5): p. 252264.

8. $\quad$ Nebel, C.E., From gemstone to semiconductor. Nature Materials, 2003. 2(7): p. 431-432.

9. Ziegler, J., SRIM/TRIM code. 2008.

10. Vandervorst, W., F. Shepherd, and R. Downing, High resolution SIMS and neutron depth profiling of boron through oxide-silicon interfaces. Journal of Vacuum Science \& Technology A: Vacuum, Surfaces, and Films, 1985. 3(3): p. 1318-1321.

11. Ziegler, J., J. Biersack, and M. Ziegler, SRIM: The Stopping and Range of Ions in Matter. 5 ed. 2015, Chester, MD: SRIM Co.

12. Chen-Mayer, H., et al., Two aspects of thin film analysis: boron profile and scattering length density profile. Nuclear Instruments and Methods in Physics Research Section A: Accelerators, Spectrometers, Detectors and Associated Equipment, 2003. 505(1-2): p. 531-535. 